\title{
Uptake of Neutrophil-Derived Ym1 Protein Distinguishes Wound Macrophages in the Absence of Interleukin-4 Signaling in Murine Wound Healing
}

\author{
Itamar Goren, Josef Pfeilschifter, and Stefan Frank
}

From the pharmazentrum frankfurt/ZAFES, Klinikum der Johann Wolfgang Goethe-Universität, Frankfurt am Main, Germany

\author{
Accepted for publication \\ August 4, 2014 \\ Address correspondence to \\ Stefan Frank, Ph.D., pharma \\ zentrum frankfurt/ZAFES, \\ Klinikum der JW Goethe- \\ Universität, Theodor-Stern-Kai \\ 7, D-60590 Frankfurt am Main, \\ Germany. E-mail: s.frank@em. \\ uni-frankfurt.de.
}

\begin{abstract}
The determination of regenerative wound-healing macrophages as alternatively activated macrophages is currently questioned by the absence of IL- 4 in wound tissue. Yet, murine wound tissue expressed high levels of Ym1 (chitinase 3-like 3), an established marker of the IL-4-induced alternatively activated macrophage phenotype. Ym1 was expressed in wound neutrophils but not in macrophages. Initially, Ym1-free woundhealing macrophages, invading from the wound margins, became gradually positive for the protein in the absence of IL-4 signaling and Stat6 activation, as they entered the neutrophil-populated wound regions. IL-4 failed to induce Ym1 protein in ex vivo-cultured wound tissue explants containing wound-healing macrophages. Recombinant Ym1 protein was selectively taken up by macrophages but not by keratinocytes and endothelial cells. Cultured macrophages lost the ability to take up the recombinant protein when four highly conserved residues and the 70-amino acid small $\alpha+\beta$ domain essential for Ym1 function were removed. The data suggest that the IL-4/Stat6-independent presence of Ym1 protein in wound-healing macrophages is of exogenous origin, with Ym1 taken up from wound neutrophils as the cellular source. The data suggest that in situ determination of wound-healing macrophages, often defined by Ym1, might not essentially describe an IL-4-dependent macrophage phenotype. Consequently, wound-healing macrophages should not be classified by the established categories of the well-accepted but simplified paradigm of M1/M2 macrophage activation. (Am J Pathol 2014, 184: 3249-3261; http://dx.doi.org/10.1016/j.ajpath.2014.08.011)
\end{abstract}

Starting in the 1970s and based on the groundbreaking work of Leibovich and colleagues ${ }^{1}$ using a guinea pig model of incisional wound healing, the concept and functional implications of the wound-healing macrophage seemed to be intriguingly simple. Using glucocorticoids or an antimacrophage serum, the authors were able to show the pivotal role of the wound-healing macrophage for normal development of granulation tissue and removal of cellular debris from the site of injury. ${ }^{1}$ However, it soon turned out that macrophages respond to different physiologic or pathologic conditions with remarkably expressed plasticity. Moreover, it is now evident that even circulating macrophage predecessors display diverse subpopulations of monocytes in the vasculature. ${ }^{2}$ Two functional subpopulations have been described among murine blood monocytes, with differential properties to be recruited into inflamed or noninflamed tissue. ${ }^{3}$ Early in tissue injury, inflammatory monocyte precursors specified as lymphocyte antigen (Ly)-6C ${ }^{(+)}, \mathrm{C}-\mathrm{C}$ chemokine receptor $2^{(+)}$, and CX3C chemokine receptor $3^{(-)}$ rapidly infiltrate sites of inflammation. ${ }^{4,5}$ Maturation of these Ly-6C $\mathrm{C}^{(+)}$and C-C chemokine receptor $2^{(+)}$monocytes into wound-healing macrophages adds expression of the mannose receptor and results in proinflammatory cytokine release (tumor necrosis factor $\alpha$ and IL-6) and phagocytosis. ${ }^{4}$ Later stages of wound tissue are then subsequently populated by woundhealing macrophages derived from Ly- $6 \mathrm{C}^{(-)}, \mathrm{C}-\mathrm{C}$ chemokine receptor $2^{(-)}$, and $\mathrm{CX} 3 \mathrm{C}$ chemokine receptor $3^{(+)}$infiltrating monocytes, which secrete factors to stimulate fibrosis (eg, transforming growth factor- $\beta 1$ ) and angiogenesis (eg, vascular endothelial growth factor). ${ }^{3,4,6}$

The concept of classically activated proinflammatory macrophage and alternatively activated repair-type macrophage phenotypes that emerge during macrophage maturation

Supported by the Deutsche Forschungsgemeinschaft (SFB 815, grant FR 1540/1-2, GK 1172 to S.F.).

Disclosures: None declared. 
under varying physiologic and pathologic conditions is now an integral part of the respective literature. ${ }^{7}$ However, the early description of wound-healing macrophages as an alternatively activated macrophage phenotype ${ }^{8}$ now seems to be an attempt to shoehorn repair-associated macrophages into the established pattern of classically or alternatively activated macrophage phenotypes. This concept is no longer supported because murine wound-healing macrophages obviously show traits of both classically and alternatively activated phenotypes in the absence of wound-derived IL-4 and IL-13, cytokines proposed to essentially induce the wound-healing phenotype in macrophages. ${ }^{2,4}$

However, the presence of arginase-1 (Arg-1) and Ym1 (chitinase 3-like 3) proteins constitutes a reliable marker of alternatively activated macrophages in mice. ${ }^{7,9-11}$ The notion to regard wound-healing macrophages as a specialized repair phenotype of an alternative macrophage activation, capable of controlling inflammation and stimulating regeneration, might be derived from the observation that murine wound-healing macrophages also express Arg-1 and Ym1 proteins. ${ }^{4,12}$ Yet, this approach seems to simplify macrophage activation in wounds, which also contradicts the IL-4-/IL-13-dependent alternative phenotype in Th2 immune response to parasites, ${ }^{13}$ as wound tissue is devoid of IL-4, IL-13, and active Stat6. ${ }^{4}$

Use of the Ym1 protein as a marker for wound-healing macrophages might again add to this confusing situation. Besides its expression in alternatively activated macrophages, ${ }^{7,9-11} \mathrm{Ym} 1$ is also present at much higher concentrations in peritoneal and bone marrow-derived neutrophils. ${ }^{14}$ Moreover, Ym1 contains a secretory signal peptide and is secreted in large amounts from the cells. ${ }^{15}$ Thus, the dynamic of $\mathrm{Ym} 1$ protein appearance obviously challenges its use as a marker to define a wound-healing macrophage phenotype. In accordance with this notion, a recent study on wound-healing macrophages used Ym1 to state that the early and inflammatory phase of repair promoted an alternative activation of macrophages at the wound site. ${ }^{12}$

However, herein we show that Ym1 is clearly associated with the appearance and localization of neutrophils in normal and impaired wound tissue. These data suggest that neutrophilreleased Ym1 protein is specifically taken up by infiltrating wound-healing macrophages. Importantly, wound-healing macrophages amid the granulation tissue could not be induced to express Ym1 protein by IL-4 stimulation as a result of skin-specific suppression of this particular signaling pathway. Thus, the localization of $\mathrm{Ym} 1$ in wound-healing macrophages only mimics an alternative activation of the cells in the absence of an IL-4/Stat6 signaling network in wounds.

\section{Materials and Methods}

\section{Reagents}

Phenylmethylsulfonyl fluoride, dithiothreitol, aprotinin, sodium fluoride, sodium orthovanadate, and EDTA were from Sigma-Aldrich Chemie GmbH (Taufkirchen,
Germany). Leupeptin and ocadaic acid were from BIOTREND Chemikalien GmbH (Cologne, Germany). Deoxynucleoside triphosphate, random hexamers, and DNA restriction endonucleases were obtained from Roche $\mathrm{Di}$ agnostics GmbH (Mannheim, Germany). Taq- and Pfu polymerase were purchased from Promega GmbH (Mannheim, Germany). Purified recombinant mouse cytokines were from PeproTech Inc. (Tebu-bio, Offenbach, Germany).

\section{Antibodies}

Rat anti-Ly-6G and anti-Ly-6C [granulocyte antigen-1 (Gr1)] (553123; BD Biosciences, Heidelberg, Germany), rat antimouse F4/80 (MCA497; AbD Serotec, Düsseldorf, Germany), rat anti-mouse neutrophils (Ly-6B2, clone7/4) (MCA771; AbD Serotec), rat anti-mouse CD16/CD32 (553142; BD Pharmingen, Heidelberg, Germany), phycoerythrin-conjugated rat antimouse Ly-6G (551461; BD Pharmingen), PerCP-Cy 5.5 rat anti-mouse Ly-6C (560525; BD Pharmingen), rabbit antiArg-1 (generated by the authors and raised against peptide $\mathrm{H}_{2} \mathrm{~N}-\mathrm{CEGNHKPETDYLKPPK-COOH}$ ), rabbit anti-Ym1 (01404; STEMCELL Technologies SARL, Cologne, Germany), rabbit anti-resistin-like molecule $\alpha$ (ab39626; Abcam, Quartett, Berlin, Germany), rabbit anti-cyclooxygenase-2 (Cayman 160126; via Biozol, Eching, Germany), rabbit antinitric oxide synthase-2 (sc-650; Santa Cruz Biotechnology, Heidelberg, Germany), rabbit monoclonal anti-Stat6 (YE361, Epitomics; via Biomol GmbH, Hamburg, Germany), rabbit anti-p-Stat6 (Y-641) (sc-11762-R; Santa Cruz Biotechnology), rabbit anti-c-Myc (C3956; Sigma-Aldrich Chemie $\mathrm{GmbH}$ ), rabbit monoclonal anti-Flag (F2555; Sigma-Aldrich Chemie GmbH), mouse monoclonal anti-Flag (F3165; Sigma-Aldrich Chemie $\mathrm{GmbH}$ ), mouse monoclonal anti$\beta$-actin (A5441; Sigma-Aldrich Chemie GmbH), nonspecific rat IgG (Santa Cruz Biotechnology), fluorescein isothiocyanatelabeled goat anti-rabbit (11-4839; eBioscience, Frankfurt, Germany), fluorescein isothiocyanate-conjugated rabbit IgG isotype antibody (11-4614; eBioscience), and rabbit anti-glyceraldehyde-3-phosphate dehydrogenase (GAPDH) (2275-Pc; Trevigen, Frankfurt, Germany).

\section{Animals}

Female C57B1/6J (wild-type), leptin-deficient (C57B1/6Jobese/obese), and $\mathrm{Stat}^{-1-}$ C57B1/6 (B6.129S2-Stat6 $\left.{ }^{\text {tmlGru }} / \mathrm{J}\right)$ mice were obtained from Charles River Laboratories (Sulzfeld, Germany). At age 10 weeks, the mice were caged individually, monitored for body weight, and wounded as described in the following subsection.

\section{Wounding of Mice}

Wounding of mice was performed as described previously. ${ }^{16,17}$ Briefly, mice were anesthetized. Subsequently, six full-thickness wounds ( $5 \mathrm{~mm}$ in diameter, 3 to $4 \mathrm{~mm}$ apart) were made on the backs of the mice by excising the skin 
and the underlying panniculus carnosus. The wounds were allowed to form a scab. An area of 7 to $8 \mathrm{~mm}$ in diameter, which included the granulation tissue and the complete epithelial margins, was excised at the indicated time points for analysis. Back skin from nonwounded mice served as a control. Twelve wounds isolated from four animals were used for RNA analysis. For immunoblot analysis, eight wounds from four individual mice were used. All the animal experiments were performed according to the guidelines and approval of the local ethics animal review board (Regierungspräsidium Hessen, Darmstadt, Germany).

\section{Treatment of Mice}

To induce a systemic depletion of neutrophils (neutropenia), purified rat monoclonal anti-neutrophil antibody Ly-6G/Ly6C $(\mathrm{Gr}-1)^{18}(1 \mathrm{mg} / \mathrm{g}$ body weight) was injected i.p. in $0.5 \mathrm{~mL}$ of phosphate-buffered saline (PBS) 24 hours before wounding into $\mathrm{C} 57 \mathrm{Bl} / 6 \mathrm{~J}$ wild-type mice. A nonspecific rat IgG served as control. $o b / o b$ mice were treated i.p. by PBS or leptin $(2 \mu \mathrm{g} / \mathrm{g}$ body weight) during wound healing as described in detail previously. ${ }^{19,20}$

\section{Ex Vivo Analysis of Skin and Lung Tissue from Nonwounded and Wounded Mice}

Skin and lung tissues were isolated under sterile conditions from nonwounded and wounded mice (3 days after injury). Pieces of tissue $(3 \times 3 \times 3 \mathrm{~mm})$ were subsequently cultured ex vivo in RPMI 1640 medium with or without IL-4 (10 ng/ $\mathrm{mL}$ ) supplementation for the indicated periods. The tissue samples were directly fixed in formalin $(4.5 \%$ buffered in PBS) or zinc fixative (see later in the Immunohistochemical Analysis section) for immunohistochemical analysis or were snap frozen for RNA and protein preparation.

\section{Thioglycollate-Elicited Peritoneal Macrophages}

Mice were injected i.p. with a single injection of $0.5 \mathrm{~mL}$ of a sterile thioglycollate solution $\left(8 \% \mathrm{w} / \mathrm{v}\right.$ in $\mathrm{H}_{2} \mathrm{O}$ ) (SigmaAldrich Chemie $\mathrm{GmbH}$ ). Five days after injection, mice were sacrificed and peritoneal cells were isolated by peritoneal lavage using RPMI 1640 medium. Erythrocytes were lyzed in ACK buffer $\left(150 \mathrm{mmol} / \mathrm{L} \mathrm{NH}_{4} \mathrm{Cl}, 10 \mathrm{mmol} / \mathrm{L} \mathrm{KHCO}_{3}\right.$, and $0.1 \mathrm{mmol} / \mathrm{L} \mathrm{Na}_{2} \mathrm{EDTA}$ ), and the remaining leukocytes were collected by centrifugation $(250 \times g)$. Cells $\left(3 \times 10^{6}\right.$ per well $)$ were seeded in 6-well plates using RPMI 1640 medium and were incubated for an additional 16 to 20 hours before stimulation.

\section{Bone Marrow-Derived Macrophages}

Isolated femora and tibiae from nonwounded and wounded mice (see Wounding of Mice) were flushed out using RPMI 1640 medium to obtain bone marrow cells for culturing. Flushed cells were collected in RPMI 1640 medium by centrifugation $(250 \times g)$. After lysis of erythrocytes in $0.83 \%$ $\mathrm{NH}_{4} \mathrm{Cl}$, the remaining cells were analyzed by fluorescenceactivated cell sorting (see Flow Cytometry) or cultured in Dulbecco's modified Eagle's medium/F12 medium (\#11039; Gibco Life Technologies, Darmstadt, Germany) in the presence of macrophage colony-stimulating factor $(1 \mathrm{ng} / \mathrm{mL})$ at $3.5 \times 10^{5}$ cells $/ \mathrm{cm}^{2}$ for 8 to 10 days to obtain adherent cell fractions for further studies.

\section{Flow Cytometry}

Isolated bone marrow cells from nonwounded and injured mice (see Wounding of Mice) were recovered in fluorescenceactivated cell sorting staining buffer [ $2 \%$ fetal calf serum $(\mathrm{v} / \mathrm{v})$ and $0.1 \% \mathrm{Na}_{3} \mathrm{~N}(\mathrm{w} / \mathrm{v})$ in PBS]. Cells $\left(2 \times 10^{6}\right)$ were blocked with rat anti-mouse CD16/CD32 and were stained for Ly-6G using phycoerythin-conjugated rat anti-mouse Ly-6G as previously described. ${ }^{20}$ Cells were fixed and permeabilized using the BD Cytofix/Cytoperm kit (554714; BD Biosciences). Rabbit anti-Ym1 and fluorescein isothiocyanate-labeled goat anti-rabbit were used to detect $\mathrm{Ym} 1$ protein. Intracellular staining was controlled using a fluorescein isothiocyanateconjugated rabbit IgG isotype antibody (11-4614; eBioscience). Labeled cells were analyzed using a FACSCantoII flow cytometer (BD Biosciences).

\section{Cell Culture}

The murine RAW 264.7 macrophage cell line was cultured in RPMI 1640 medium. The murine brain endothelial cell (BND3) and the human keratinocyte cell line $\mathrm{HaCaT}^{21}$ were cultured in Dulbecco's modified Eagle's medium. Dulbecco's modified Eagle's medium, RPMI 1640 medium, penicillin/streptomycin, and $0.05 \%$ Trypsin-EDTA were purchased from Gibco Life Technologies. Fetal bovine serum was obtained from Biochrom (Berlin, Germany).

\section{RNA Isolation and RNase Protection Analysis}

RNA isolation and RNase protection assays were performed as described previously. ${ }^{17,22}$ If not indicated otherwise, every experimental time point depicts 12 wounds isolated from four individual mice for all RNase protection assays analyzing wound tissues samples. All the samples were quantified using phosphorimager PSL counts per $15 \mu \mathrm{g}$ of total wound RNA. The murine cDNA probes were cloned using RT-PCR. The probes corresponded to nucleotides 1174 to 1441 [for Ym1 (chitinase 3-like 3); GenBank (http://www.ncbi.nlm.nih.gov/genbank; Accession number NM_009892.2)] or to nucleotides 163 to 317 [for murine GAPDH; GenBank (http://www.ncbi.nlm.nih.gov/genbank; Accession number NM_008084.2)] of the published sequences. For RNA protection assays, $1000 \mathrm{cpm}$ of the hybridization probe was used as a size marker. tRNA hybridization showed the specificity of the probe. Hybridization against $G A P D H$ was used as a loading control. 


\section{RT-PCR}

RT-PCR was performed to assess the expression of Ym1 in isolated peritoneal macrophages. One microgram of total RNA was transcribed using random hexameric primers and Moloney murine leukemia virus reverse transcriptase (Promega $\mathrm{GmbH}$ ) according to the manufacturer's instructions. For Ym1, $5^{\prime}$-GATCTCAATATACACAGTGC-3' (forward) and $5^{\prime}$ GAGCTTAGCCAAAGCTGAC-3' (reverse) and for GAPDH, $5^{\prime}$-CTGGCATTGCTCTCAATGAC-3' (forward) and $5^{\prime}$ TCTTACTCCTTGGAGGCC-3' (reverse), primers were used to amplify the murine sequences. PCR was performed using a T3000 thermocycler (Biometra, Göttingen, Germany).

\section{Real-Time Quantitative PCR}

Real-time quantitative PCR (qPCR) was performed to assess the expression of genes of interest. TaqMan fast advanced master mix (cat. No. 4444557) and the predesigned qPCR assays were purchased from Applied Biosystems (Darmstadt, Germany): Ym1 (chitinase 3-like 3) (Mm00657889-mH), resistin-like molecule $\alpha$ (Mm00445109-m1), Arg-1 (Mm00475988-m1), lipocalin-2 (Mm01324470-m1), EGF-like module-containing mucin-like hormone receptor-like 1 (Mm00802529-m1), or 4352339E for GAPDH. qPCR was performed using a 7500 fast real-time PCR system (Applied Biosystems, Heidelberg, Germany) as follows: $95^{\circ} \mathrm{C}$ (20 seconds), 40 cycles; $95^{\circ} \mathrm{C}$ (3 seconds) and $60^{\circ} \mathrm{C}$ (30 seconds). Analyses of qPCR runs were performed using Sequence Detector software.

\section{Preparation of Protein Lysates and Western Blot Analysis}

Skin, lung, and wound tissue biopsy samples or cultured cells were homogenized in lysis buffer [1\% Triton X-100 (Roche Diagnostics GmbH), 20 mmol/L Tris/ $\mathrm{HCl}$ (pH 8.0), $137 \mathrm{mmol} /$ $\mathrm{L} \mathrm{NaCl}, 10 \%$ glycerol, $1 \mathrm{mmol} / \mathrm{L}$ dithiothreitol, $5 \mathrm{mmol} / \mathrm{L}$ EDTA, $10 \mathrm{mmol} / \mathrm{L}$ sodium fluoride, $2 \mathrm{mmol} / \mathrm{L}$ sodium orthovanadate, $1 \mathrm{mmol} / \mathrm{L}$ phenylmethylsulfonyl fluoride, $5 \mathrm{ng} / \mathrm{mL}$ aprotinin, $5 \mathrm{ng} / \mathrm{mL}$ leupeptin, and $50 \mathrm{nmol} / \mathrm{L}$ okadaic acid]. Extracts were cleared by centrifugation. Protein concentrations were determined using the BCA protein assay kit (Thermo Scientific, Cologne, Germany). Total protein (25 to $50 \mu \mathrm{g}$ ) was analyzed by SDS gel electrophoresis. After transfer to a nitrocellulose membrane, specific proteins were detected using primary antibodies. A secondary antibody against goat, rabbit, or mouse IgG coupled with horseradish peroxidase (Bio-Rad Laboratories $\mathrm{GmbH}$, Munich, Germany) and the enhanced chemiluminescence detection system (Amersham, Freiburg, Germany) were used to visualize the proteins.

\section{Enzyme-Linked Immunosorbent Assay}

Quantification of murine IL-4 was performed using the murine Quantikine enzyme-linked immunosorbent assay (ELISA) kit (R\&D Systems, Wiesbaden, Germany) according to the manufacturer's instructions.
Immunohistochemical Analysis

Skin biopsy samples were isolated from the back, fixed in formalin or in zinc fixative solution $(0.05 \%$ calcium acetate. $2 \mathrm{H}_{2} \mathrm{O}, 0.5 \%$ zine acetate. $2 \mathrm{H}_{2} \mathrm{O}, 0.5 \%$ zinc chloride in 0.1 $\mathrm{mol} / \mathrm{L}$ Tris- $\mathrm{Cl}, \mathrm{pH}$ 7.4), and embedded in paraffin. Fourmicrometer sections were counterstained with hematoxylin and eosin. For immunostaining, sections were incubated overnight at $4{ }^{\circ} \mathrm{C}$ with antibodies raised against mouse neutrophils (Ly-6B2), F4/80, or Ym1. Primary antibodies were detected using a biotinylated secondary antibody. The sections were subsequently stained with the avidin-biotin-peroxidase complex system (Santa Cruz Biotechnology) using 3,3diaminobenzidine-tetra-hydrochloride or Fast Red substratechromogen system (Dako, Hamburg, Germany) as a chromogenic substrate. Finally, sections were counterstained with hematoxylin and were mounted.

\section{Immunofluorescence}

RAW 264.7 macrophages were grown on glass slides and incubated with supernatants from pCMV-mmYm1F stably transfected human embryonic kidney (HEK) 293 cells for 24 hours. Supernatants from mock-transfected HEK 293 cells served as controls. RAW cells were fixed using $2 \%$ paraformaldehyde for 15 minutes and were subsequently blocked with $5 \%$ goat serum diluted in PBS/Triton $(0.1 \% \mathrm{w} / \mathrm{v})$. A rabbit monoclonal anti-Flag antibody (Sigma-Aldrich Chemie $\mathrm{GmbH}$ ) was incubated for 1 hour at room temperature. The fluorescencecoupled secondary antibody (Alexa Fluor 488; Molecular Probes, Leiden, the Netherlands) was diluted (1:250) in 5\% goat serum/PBS and incubated in the dark for 30 minutes. Nuclei were counterstained using DAPI (Sigma-Aldrich Chemie GmbH).

\section{Expression and Purification of Recombinant Ym1 Wild-Type and Mutated Proteins}

A full-length mouse $Y m l$ open reading frame was amplified from total day 1 wound RNA using RT-PCR. Ym1 forward primer $5^{\prime}$-CGATGTCGACCCACCATGGCCAAGCTCATTCTTG- $3^{\prime}$ and reverse primer 5'-GCATGGATCCATAAGGGCCCTTGCAACTTG-3' were used. Amplicons were digested with SalI and BamHI and were cloned into XhoI/ BamHI sites of pCMV-FLAG-N3. ${ }^{23}$ The resulting pCMVmmYm1F vector was used to express a recombinant Flagtagged Ym1 wild-type protein. The pCMV-mmYm1F vector was used as a template to generate a Ym1-Myc-tagged construct. 5'-GCATGGTACCGCTAGCCCACCATGGCCAAGCTCATTCTTG-3' and 5'-GAATGGTGACCATAAGGGCCCTTGCAACTTGC- $3^{\prime}$ were used as primers. Amplicons were cloned via $\mathrm{KpnI} / \mathrm{BstEII}$ sites into pcDNA3.1-myc-HisC (Invitrogen, Karlsruhe, Germany) to finally generate a Ym1-Myc-six histidine (6xHis)-tagged expression plasmid (pcYm1-Myc-6xHisC).

Mutation of specific amino acid residues in the $\mathrm{Yml}$ sequence residues was performed using the pCMV-mmYm1F 

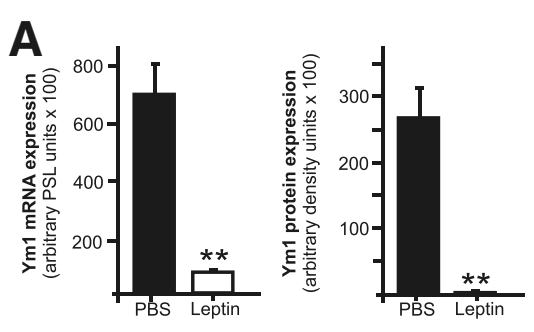

B

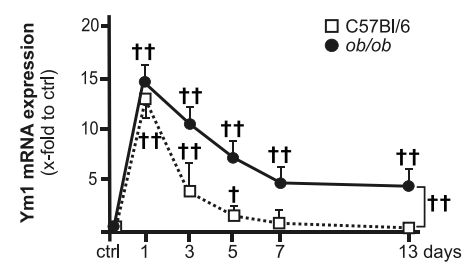

D

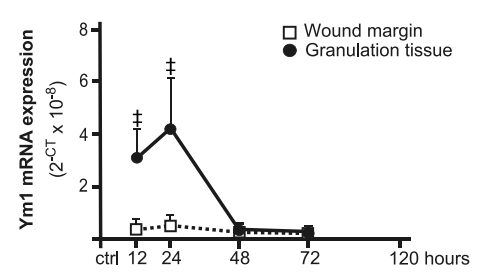

E

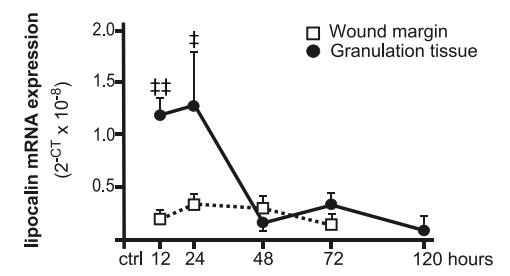

C

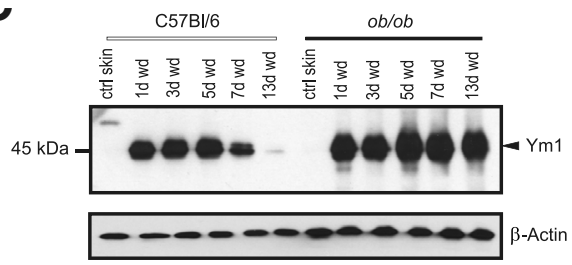

F

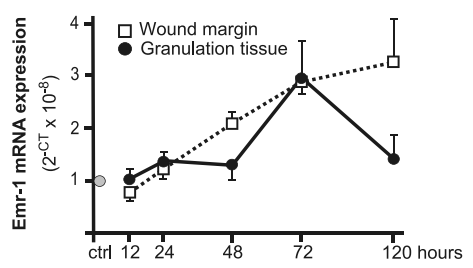

G

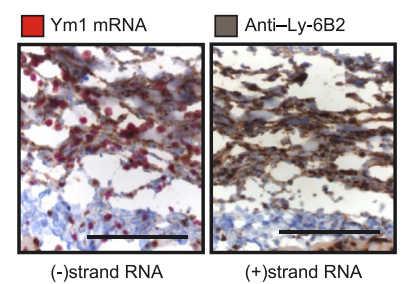

H

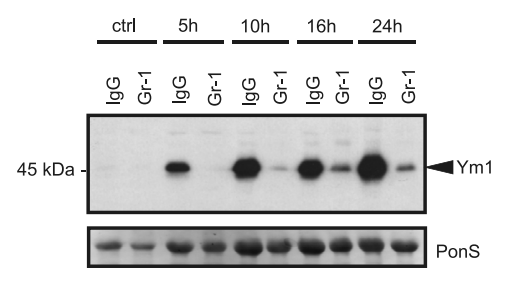

Figure 1 Ym1 expression in wounds. A: Wound tissue (13 days) of PBS- or leptin-treated diabetic ob/ob mice was analyzed for Ym1 mRNA by RNase protection assay or immunoblot for Ym1 protein expression in mice as indicated. Values were obtained from 12 wounds (nine mRNA and three protein) isolated from three animals. B: Analysis of Ym1 mRNA expression by RNase protection assay from skin and wound tissue at different time points in wild-type or diabetic $o b / o b$ mice as indicated. Values were obtained from three independent experimental series representing 36 total wounds isolated from nine animals. C: Expression of Ym1 protein in skin and wound tissue at different time points in wild-type or diabetic $o b / o b$ mice as indicated. $\beta$-Actin was used as a loading control. Real-time quantitative PCR quantification of Ym1 (D), lipocalin (E), and EGF-like module-containing mucin-like hormone receptor-like (Emr) 1 (F) mRNA in skin (ctrl), or wound margin and wound granulation tissue in wild-type mice. The time points after injury are indicated. Values were obtained from 12 wounds isolated from four animals. G: Double staining of 1-day wound tissue for Ym1 mRNA (in situ hybridization, red signals) and neutrophil-specific Ly-6G/Gr-1 (immunohistochemical analysis, brown signals). The $\mathrm{Ym}_{1}{ }^{(+)}$strand probe served as a negative control. $\mathrm{H}$ : Immunoblot showed $\mathrm{Ym} 1$ protein in the presence (IgG) or absence $(\mathrm{Gr}-1)$ of neutrophils in wound tissue at the indicated time points on injury. Ponceau $S$ (PonS) staining was used to control equal loading. Data are given as means \pm SD. ${ }^{* *} P<0.01$ (Student's unpaired $t$-test) compared with PBS-treated mice (A). ${ }^{\dagger} P<0.05,{ }^{\dagger \dagger} P<0.01$ (analysis of variance, Dunnett method) compared with nonwounded control skin (ctrl) (B). ${ }^{\ddagger} P<0.05,{ }^{\ddagger \ddagger} P<0.01$ (Student's unpaired $t$-test) compared with wound margin (D and $\mathbf{E}$ ). Scale bar $=50 \mu \mathrm{m}(\mathbf{G})$.

vector as a template (QuikChange site-directed mutagenesis kit; Stratagene, Heidelberg, Germany) and the following primers (underlined letters determine mutated or changed nucleotides): 5'-TAACTTTGATGGCCTCGCĆCTGGCC̣TGGGGGTACCCTGGGTCTCG- $3^{\prime}$ and $5^{\prime}$-CGAGA $\bar{C} C \bar{C} A G G G T \overline{A C C C}-$ CCAGGCCAGGGCGAGGCCATCAAAGTTA-3' (for mutation of N136 to A, D138 to A, and Q140 to G) or 5'CAGGTCATGACATATGCTCTCCATGATCCTAAG-3' and $5^{\prime}$-CTTAGGATCATGGAGAGCATATGTCATGACCTG-3' (for mutation of D213 to A). The resulting plasmid is referred to as pCMV-Ym1-N* $D^{*} Q^{*} D^{*}-213 F$. Deletion of the small $\alpha+\beta$ domain $^{24}$ was performed via PCR-directed DNA depletion using 5'-CTTGAAGCTCCTGACATTGTCATATGCTGGAAATCCCACAATGAG- ${ }^{\prime}$ and $5^{\prime}$-CTCATTGTGGGATTTCCAGCATATGACAATGTCAGGAGCTTCAAG- $3^{\prime}$ together with the $\mathrm{Ym} 1$ forward and reverse primers (see $R T-P C R)$ on the pCMV-Ym1-N"D*Q* ${ }^{*}-213 F$ plasmid. The mutated open reading frame was cloned into $\mathrm{Xhol} / \mathrm{BamHI}$ sites of pCMV-FLAG-N3 ${ }^{23}$ to generate pCMV-Ym1- $\Delta S$ N*D*Q*'-213F. The Nhel/BamHI-excised Ym1- $\Delta$ S$\mathrm{N}^{*} \mathrm{D}^{*} \mathrm{Q}^{*} \mathrm{D}^{*} 213 \mathrm{~F}$ open reading frame was then cloned to a $6 \mathrm{xHis}$ linker (5'-GATCCCACCATCACCATCACCATTGAT- $3^{\prime}$ ) and into the NheI/XbaI sites of the pcDNA3.1 vector (Invitrogen) to finally generate pc-Ym1- $\Delta$ S-N* ${ }^{*} Q^{*} D^{*} 213-6 x H i s$.

\section{Expression and Purification of Recombinant Ym1 Protein}

The HEK 293 cell line was stably transfected with the plasmids pcYm1-Myc-6xHisC, pc-Ym1- $\Delta$ S-N*D*Q* D*213-6xHis, and pCMV-mmYm1F. The respective cell lines secreted Myc6xHis-tagged Ym1, $\Delta$ S-N*D*Q* D*213-6xHis-tagged Ym1, and Ym1-Flag-tagged protein into the cell culture supernatants. 6xHis-tagged Ym1 proteins were purified using Ni-NTA agarose (Qiagen, Hilden, Germany). Potential endotoxin contaminations in purified protein samples were removed using an 

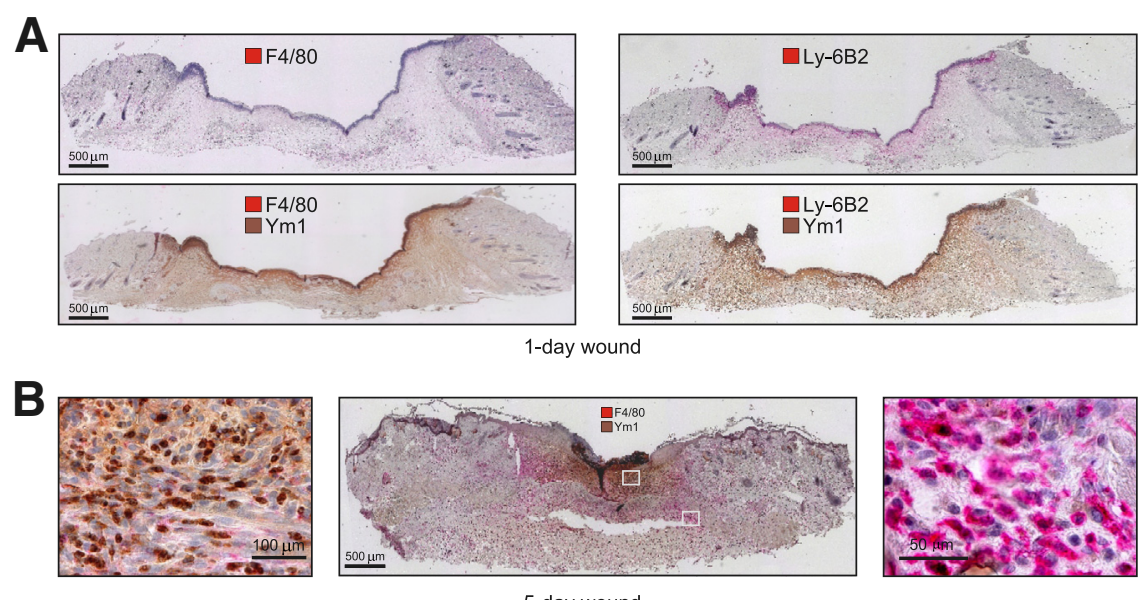

C

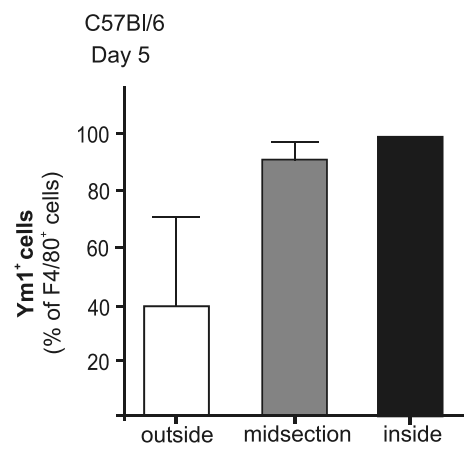

D

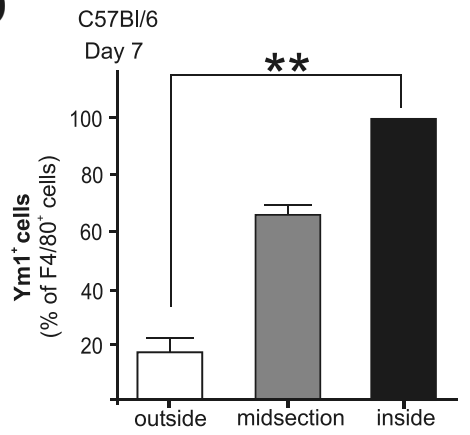

Figure 2 Localization of $\mathrm{Ym}_{1}{ }^{(+)}$woundhealing macrophages in wounds. A: Sections from 1-day wounds isolated from wild-type mice were single stained using antibodies directed against macrophage-specific $F 4 / 80$ or neutrophilspecific Ly-6B2 (clone 7/4) (red signals) or double stained by the additional use of an antiYm1 antibody (brown signals) as indicated. B: Five-day wounds were double stained using macrophage-specific $F 4 / 80$ (red) and anti-Ym1 (brown) antibodies. The white boxes indicate the localizations of the respective magnifications. Quantification of $\mathrm{Ym}_{1}^{(+)}$wound-healing macrophages in the outer (outside), middle (midsection), and innermost (inside) parts of 5-day (C) and 7-day (D) wound tissue from wild-type mice. $\mathrm{Ym}^{(+)}$cells are given as percentage of total $\mathrm{F} 4 / 80^{(+)}$cells. The analyzed areas were $250 \mu \mathrm{m}^{2}$ in size. Bars indicate means \pm SD. Values were obtained from three wounds isolated from three animals. ${ }^{* *} P<0.01$ (Student's unpaired $t$-test). immobilized polymyxin B Detoxi-Gel filtration endotoxinremoving column (Thermo Scientific). Protein concentrations were determined using the Pierce BCA protein assay kit (Thermo Scientific). The purity of each protein fraction was determined by SDS-PAGE and silver staining.

\section{Densitometric Analysis of Immunoblots}

Immunoblots were analyzed for positive signals using the density analysis Quantity One software version 4.6.8 (Bio-Rad Laboratories).

\section{Statistical Analysis}

Data are shown as means \pm SD. Data analysis was performed using the unpaired Student's $t$-test or analysis of variance (the Dunnett method) with raw data and GraphPad Prism software version 5.02 (GraphPad Software Inc., San Diego, CA).

\section{Results}

\section{Ym1 in Normal and Nonhealing Skin Wounds in Mice}

We first determined expression of the IL-4-inducible Ym1 protein as a marker to highlight the presence or absence of alternatively activated wound-healing macrophages ${ }^{7-11}$ in highly disturbed wounds of diabetic obese/obese $(o b / o b)$ mice. $^{19,25}$ On wounding, we established different healing conditions in $o b / o b$ mice by injection (i.p.) of PBS or the adipokine leptin concomitant to healing. As previously reported, ${ }^{19,25,26}$ we found highly disturbed wounds in PBStreated diabetic animals. The impaired wound tissues showed marked expression of Ym1 mRNA and protein (Figure 1A) expression. In contrast, healing of wound tissue in $o b / o b$ mice occurred during leptin supplementation. ${ }^{19,25}$ Healing wounds in $o b / o b$ mice were characterized by low Ym1 mRNA expression and the absence of Ym1 protein (Figure 1A). These findings suggest that the most-disturbed wound tissues expressed the highest levels of the wound-healing macrophage marker $\mathrm{Ym}^{7,8}$; Ym1 was reduced or even completely absent in healing tissue.

To track this conflicting finding, we next determined Ym1 expression throughout a normal and disturbed wound-healing process in healthy or diabetic $o b / o b$ mice. Again, normal repair in wild-type mice showed the rapid but transient appearance of Ym1 mRNA (Figure 1B) and protein (Figure 1C) during the early and acute phase of healing. Consistent with results from late wounds (Figure 1A), impaired wound tissue revealed a markedly prolonged and elevated presence of Ym1 mRNA (Figure 1B) and protein (Figure 1C).

\section{Ym1 Co-localizes with Neutrophils in Undisturbed} Acute Wounds

Next, we separated isolated wound tissue into wound margin (outer part of the wound) and granulation tissue (central part of 
the wound) as described previously. ${ }^{27} \mathrm{We}$ did so to localize $\mathrm{Ym} 1$ and immune cells in the respective wound compartments (Figure 1, D-F). The induction of Ym1 mRNA expression on injury (Figure 1B) was strongly restricted to the inner granulation tissue compartment (Figure 1D) and markedly coincided with the constitutively expressed neutrophil marker lipocalin ${ }^{28}$ (Figure 1E) within the first 24 to 36 hours of repair. Macrophage infiltration, as assessed by EGF-like module-containing mucin-like hormone receptor-like-1 mRNA, the transcript of the macrophage-specific marker F4/80, ${ }^{29,30}$ occurred later and did not preferentially co-localize to Ym1 mRNA expression in inner wounds (Figure 1F). The qPCR-based colocalization of $\mathrm{Ym} 1$ and neutrophils in wounds (Figure 1, D and E) was supported by combined in situ hybridization (for Ym1 mRNA) and immunohistochemical analysis [for the neutrophil surface marker Ly-6B2 (clone 7/4)]. We found distinct expression of $\mathrm{Ym} 1 \mathrm{mRNA}$ in infiltrating neutrophils in the granulation tissue (Figure 1G). The observed neutrophilcoupled Ym1 expression in early wounds could be strengthened experimentally as complement-driven depletion of wound neutrophils by targeting the surface marker Gr-1 using a systemic injection of the anti-Ly-6G/Ly-6C (Gr-1) monoclonal antibody ${ }^{18}$ nearly completely abolished the strong presence of Ym1 protein in early wounds (Figure 1H).

Staining of 1-day wound sections for F4/80 (macrophage marker), Ly-6B2 (neutrophil marker), and Ym1 antigens delineated the differential spreading of infiltrating neutrophils and macrophages into early wounds and a co-appearance of $\mathrm{Ym} 1$ protein to those wound regions particularly packed by neutrophils (Figure 2A). The colocalization of Ym1 protein and wound neutrophils notably intensified during later 5-day wound stages of repair. The Ym1-specific signals concentrated in the wound center (Figure 2B). In contrast, the whole entity of wound-located macrophages, migrating from below and from the edges into the wound site, was devoid of Ym1 protein (Figure 2B). Despite the finding of Ym1-free wound margin macrophages, we found nearly all wound center macrophages to hold strong Ym1-positive signals (Figure 2, B-D). This pattern might suggest a Ym1-based gradient that might interact with or even organize the macrophage influx during healing. In addition, data from granulocyte mobilization from the bone marrow of wounded mice again indicated neutrophils as an important cellular source of Ym1. Fluorescenceactivated cell sorting analysis of $\mathrm{Ly}-6 \mathrm{G}^{(+)}$bone marrow cells in mice showed significant mobilization of $\mathrm{Ym}^{(+)}$ neutrophils (defined by Ly-6G) on wounding (Figure 3).

\section{Wound Ym1 Induction in the Absence of IL-4-Mediated Stat6 Activation}

The gradual appearance of Ym1 in infiltrating woundhealing macrophages might be explained either as induction at the transcriptional level or possibly in conjunction with a gradual uptake of neutrophil-derived Ym1 at the wound site. Herein, we confirm data from Daley et al, ${ }^{4}$ who reported the lack of IL-4 and Stat6 activation in murine wounds, as we could not detect IL-4 protein (Figure 4A) or Stat6 (Figure 4B) activation in mouse wounds. Moreover, Ym1, a central marker of alternatively activated woundhealing macrophages, ${ }^{12}$ was independent of a Stat6mediated induction in wounds, as Stat6-deficient mice also showed marked induction of Ym1 mRNA (Figure 4C) and protein (Figure 4D) after wounding. In contrast, peritoneal macrophages from wild-type mice were responsive to IL-4 to express (Figure 4E) and secrete (Figure 4F) Ym1. The insignificant role of IL-4 in wounds is further strengthened by the robust expression of Arg-1, an additional marker of the well-established IL-4-/Stat6-mediated macrophage polarization, ${ }^{10,11}$ in Stat6 knockout mice (Figure 5). Also, the found in inflammatory zone $1 /$ resistin-like molecule $\alpha$ marker protein of IL-4-mediated alternative macrophage polarization ${ }^{9}$ was not induced at the wound site throughout the healing period (data not shown).

\section{Wound Tissue Macrophages Do Not Express Endogenous Ym1 Protein}

We next assessed the general responsiveness of wound tissue to an exogenous IL-4 stimulus. IL-4 and activated Stat6 were absent in wound tissue (Figure 4), but that did not exclude a basic responsiveness of wound tissue to IL-4. Ym1 protein could serve only as a regular marker for the alternative maturation wound-healing macrophages, when woundlocated macrophages will respond to an exogenous IL-4 signal. Ex vivo-cultured wound tissue expressed moderate levels of Ym1 mRNA on IL-4 addition (Figure 6A). Lung tissue-induced $\mathrm{Ym} 1$ expression from nonwounded mice was more sensitive to exogenously added IL-4. The Ym1 mRNA induction in lung tissue was even enhanced in wounded mice,

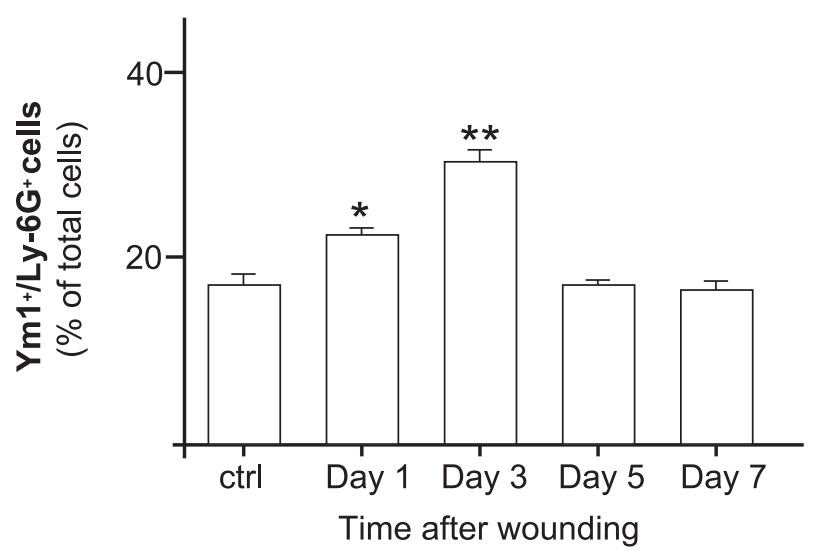

Figure 3 Mobilization of $\mathrm{Ym}_{\mathrm{m}}{ }^{(+)}$bone marrow granulocytes on skin wounding. Bone marrow cells from nonwounded (ctrl) and wounded mice were isolated. Isolated cells were analyzed for $\mathrm{Ym}_{1}{ }^{(+)}$bone marrow granulocytes by fluorescence-activated cell sorting using anti-Ly-6G and anti-Ym1 antibodies. The time points after injury are indicated. Ym1/ Ly- $6 \mathrm{G}$ - positive cells are given as percentage of total bone marrow cells. Bars indicate means \pm SD. $n=3$ animals. ${ }^{*} P<0.05,{ }^{*} P<0.01$ (Student's unpaired $t$-test) compared with ctrl. 

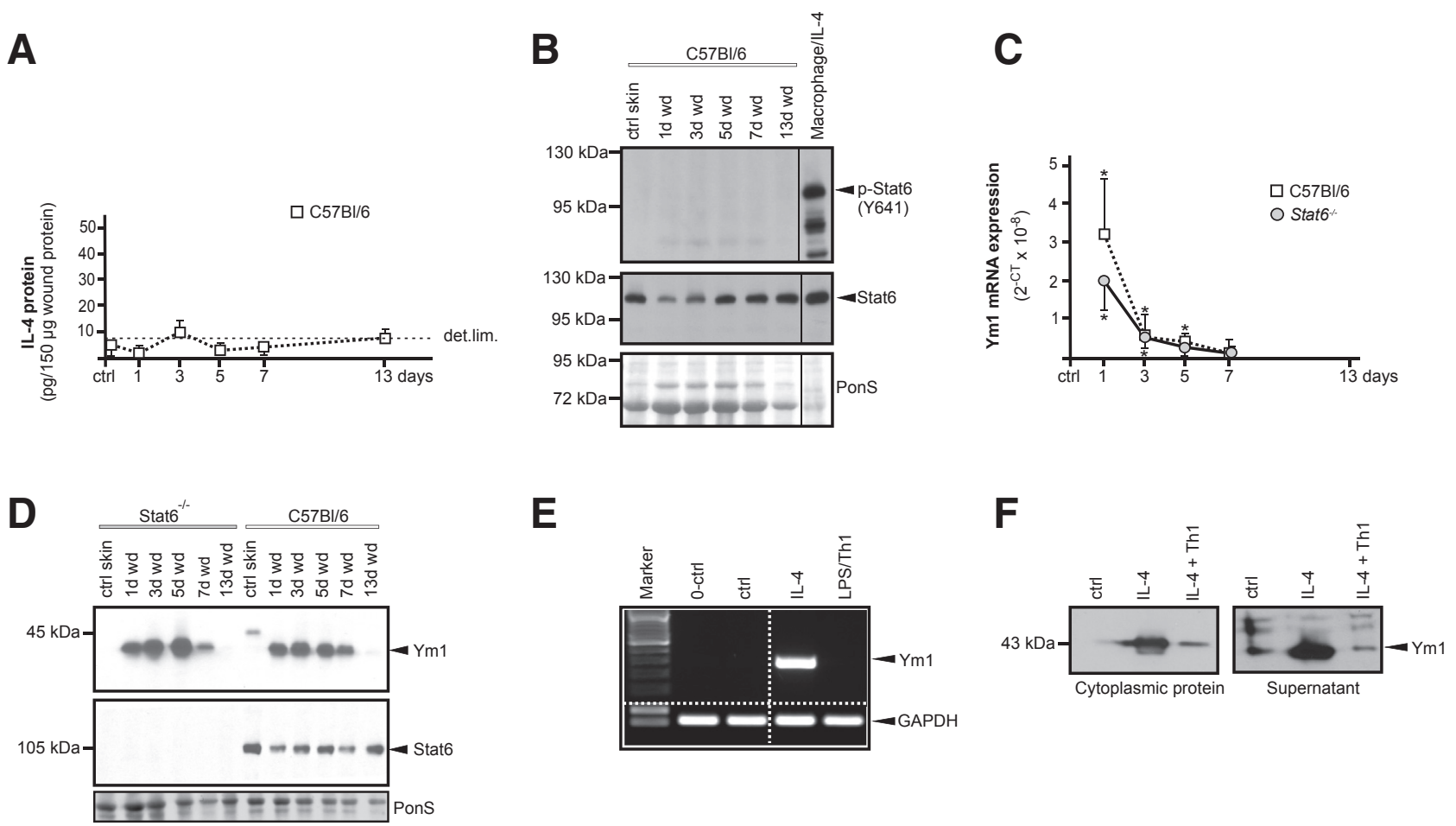

Figure 4 Ym1 expression is independent of IL-4/Stat6 signaling in wounds. A: ELISA analysis for IL-4 in lysates of skin (ctrl) and wound lysates isolated from wild-type mice at indicated time points. The line (det.lim.) demonstrates the detection limit of the assay. B: Immunoblots showing activated Stat6 (p-Stat6, Y641) and total Stat6 in skin (ctrl skin) and wounds from wild-type mice at indicated time points. IL-4-stimulated primary macrophages served as a positive control. Ponceau S (PonS) staining was used to control equal loading. C: Real-time quantitative PCR quantification of Ym1 mRNA in skin (ctrl) and during skin repair in wild-type $(\mathrm{C} 57 \mathrm{Bl} / 6)$ or Stat6-deficient $\left(\right.$ Stat $\left.^{-{ }^{-}-}\right)$mice at indicated time points. Values were obtained from 12 wounds isolated from four animals. D: Immunoblots for total Stat6 and Ym1 protein in skin (ctrl skin) and wounds from Stat $6^{-1-}$ and C57Bl/6 mice at time points as indicated. PonS staining was used to control equal loading. E: RT-PCR analysis of Ym1 mRNA expression in isolated primary peritoneal macrophages. Macrophages were left unstimulated for 24 hours (ctrl) or were treated with IL-4 $(10 \mathrm{ng} / \mathrm{mL})$ or LPS $(100 \mathrm{ng} / \mathrm{mL}) /$ Th1 $(20 \mathrm{ng} / \mathrm{mL}$ of interferon $\gamma, 25 \mathrm{ng} / \mathrm{mL}$ of tumor necrosis factor $\alpha)$ stimuli for 24 hours. Macrophages before stimulation served as an additional control (0-ctrl). F: Primary macrophages were stimulated with IL-4 or with IL-4 and Th1 or were left untreated (ctrl) for 24 hours. Fifty micrograms of total cellular protein lysate (left panel) or $25 \mu \mathrm{L}$ of the respective conditioned cell culture supernatant (right panel) was analyzed for the expression of Ym1. Bars indicate means \pm SD (A and $\mathbf{B}) . n=3$ animals $(\mathbf{A})$. ${ }^{*} P<0.05$ (analysis of variance, Dunnett method) compared with ctrl (C).

suggesting a systemic effect of skin wounding on internal organs (Figure 6A). In addition, we controlled the general sensitivity of macrophages to IL-4 from the respective animals. Isolated peritoneal and bone marrow macrophages showed elevated Ym1 mRNA levels on IL-4 stimulation (Figure 6B). Nevertheless, endogenous Ym1 protein expression, which seems critical to the alternatively activated woundhealing macrophage paradigm, did not subsequently follow its observed mRNA patterns in IL-4-stimulated wound and lung tissue. We could not find $\mathrm{Yml}^{(+)}$wound-healing macrophages in an IL-4-supplemented wound environment (Figure 6C). In contrast, lung tissue macrophages from the same individual animal expressed $\mathrm{Ym} 1$ protein on IL-4 stimulation (Figure 6D).

Biochemical analysis confirmed the absence of $\mathrm{Ym} 1$ protein in exvivo-cultured skin tissue (Figure 7A) in the presence of transient Stat6 activation (Figure 7C). Importantly, the strong Ym1 protein signals in cultured wound tissue (Figure 7B) were completely independent of IL-4-stimulated Stat6 activation in the respective tissue (Figure 7D). The Stat6independent Ym1 protein in skin wounds (Figure 7B) might, therefore, be allocated to the neutrophil subset in the cultured wound tissue. In accordance with the IL-4-induced $\mathrm{Yml}^{(+)}$ macrophages in lung tissue (Figure 6D), Ym1 protein increased over time in IL-4-treated lung tissue from normal and injured mice (Figure 7, A and $\mathrm{B}$ ) in the presence of persistent Stat6 activation (Figure 7, C and D).

\section{Macrophages Take Up Ym1 Protein}

This study suggested the presence of Ym1 protein in woundhealing macrophages independent of its endogenous induction by IL-4. ${ }^{9,11}$ To assess potential Ym1 uptake into macrophages, we first expressed a recombinant Flag-tagged Ym1 protein in transfected HEK cells from which the recombinant protein was secreted (Figure 8A). RAW 264.7 macrophages, but not HaCaT keratinocytes and BND3 endothelial cells, took up the Flag-tagged $\mathrm{Ym} 1$ protein from HEK supernatants (Figure 8, B and C). We excluded that the Flag-tagged Ym1 protein induced the expression of endogenous Ym1 in macrophages because primary and RAW 264.7 macrophages did not express endogenous Ym1 mRNA 
A

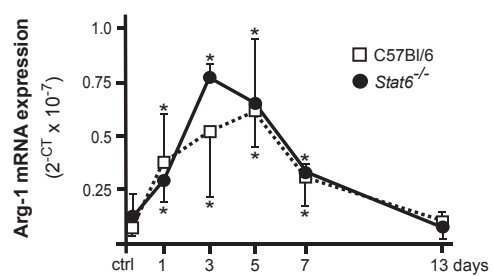

B

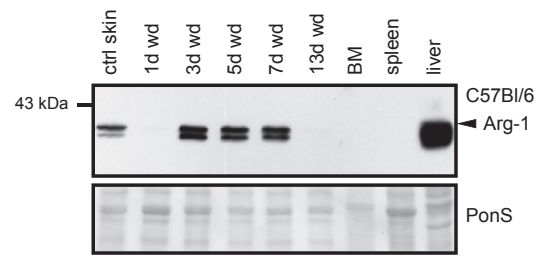

C

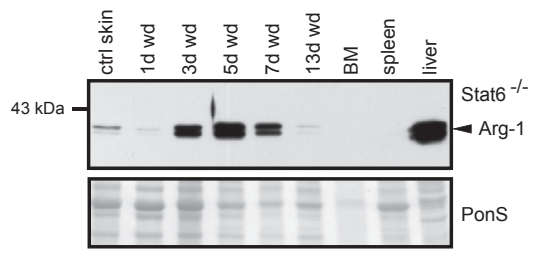

Figure 5 Arg-1 expression is independent of IL-4/Stat6 signaling in wounds. A: Real-time quantitative PCR quantification of Arg-1 mRNA expression in skin (ctrl) and during healing in wild-type $(\mathrm{C57Bl} / 6)$ or Stat6-deficient $\left(\right.$ Stat $\left.6^{-/-}\right)$mice at indicated time points. Values were obtained from 12 wounds isolated from four animals. Immunoblots for Arg-1 protein in skin (ctrl skin) and wounds from wild-type (C57Bl/6) (B) or Stat6-deficient (Stat6 ${ }^{-1-}$ ) (C) mice at indicated time points. Ponceau S (PonS) staining was used to control equal loading. Bars indicate the means \pm SD (A). ${ }^{*} P<0.05$ (analysis of variance, Dunnett method) compared with nonwounded control skin (ctrl) (A). BM, bone marrow.

in the presence of exogenously applied Flag-tagged Ym1 protein (Figure 8D).

To assess a specific uptake of Ym1 into macrophages, we mutated the Ym1 protein by replacement of essential amino acid residues (D136 to A, D138 to A, Q140 to A, and D213 to A) and by depletion of the small $\alpha+\beta$ domain $(\Delta S)$, all pivotal to three-dimensional structure and protein function. ${ }^{24}$ Finally, we added an in-frame 1.2-kDa Myc-tag for protein detection and $6 x \mathrm{xH}$ for protein purification. The resulting Ym1$N^{*} D^{*} Q^{*} D^{*}-\Delta$ S-H6-mutated protein is shown in Figure 8E. A Myc-tagged wild-type Ym1 protein served as control. Notably, the purified mutant Ym1 protein was not taken up by primary macrophages, whereas the purified wild-type Ym1 protein was effectively assimilated by the cells (Figure 8F). Moreover, the absorbed wild-type Ym1 protein interfered with an exogenously triggered low-grade inflammatory response of the cells: the Myc-tagged Ym1 protein was associated with a marked down-regulation of inducible nitric oxide synthase and cyclooxygenase-2 expression in response to low amounts of lipopolysaccharide (LPS) (Figure 8G).

\section{Discussion}

Since the first studies on macrophage function in wound healing, ${ }^{1}$ it is now beyond question that macrophages are pivotally involved in the control of tissue repair. Depletion of macrophages from wounds generated important knowledge on macrophage function in the control of wound neutrophil infiltration, inflammation, epithelialization, vascularization, and contraction. ${ }^{1,12,20,31,32}$ A recent review on wound macrophages as key regulators of repair ${ }^{2}$ now placed emphasis on the request to reexamine the phenotype of wound-healing macrophages and their role in tissue repair. This review was based on a report that had assessed the phenotype of wound-healing macrophages. ${ }^{4}$ This report questioned the well-accepted pattern of macrophage maturation
A

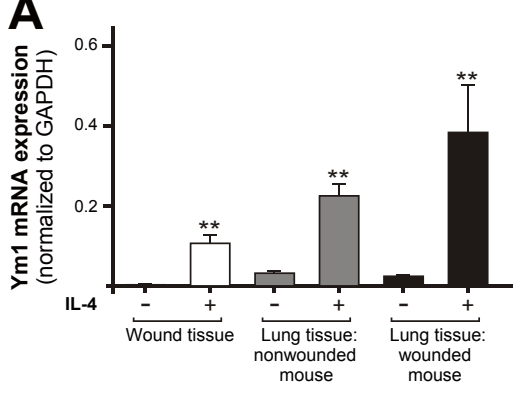

C

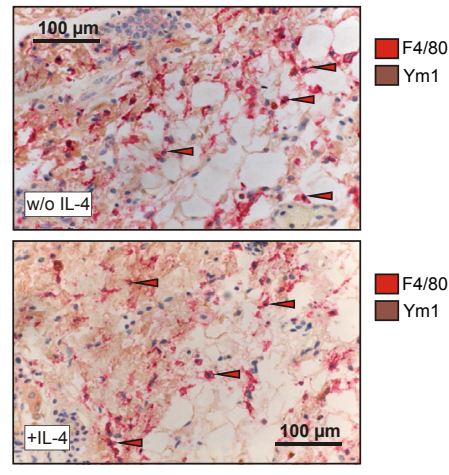

Wound tissue (day 3 )
B

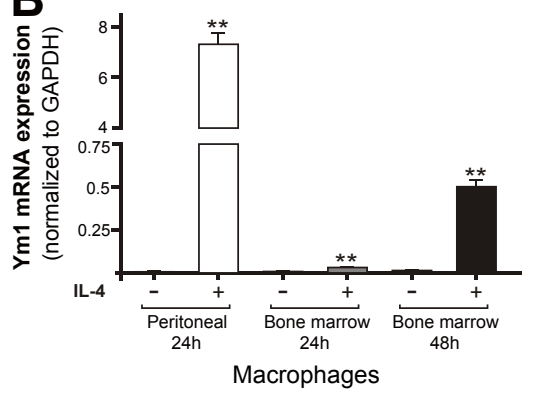

D

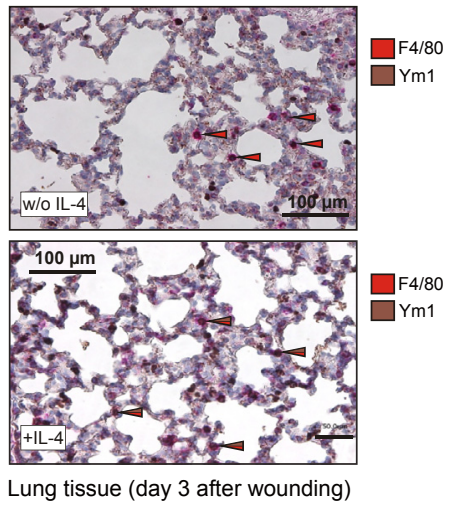

Figure 6 IL-4 does not induce Ym1 protein expression in wound macrophages. Wild-type mice were wounded. Wound and lung tissues were isolated from the animals 3 days after injury. In addition, lung tissue was isolated from nonwounded wild-type mice. The isolated tissues were cultured ex vivo in the absence or presence of 10 $\mathrm{ng} / \mathrm{mL}$ of IL-4 for 24 hours. A: Real-time quantitative PCR quantification of Ym1 mRNA expression in ex vivo wound tissue and lung tissue from nonwounded and wounded mice on IL-4 stimulation. B: Isolated peritoneal and bone marrow macrophages were cultured for the indicated periods in the absence or presence of $10 \mathrm{ng} /$ $\mathrm{mL}$ of IL-4. Values were obtained from ex vivo tissue or cells isolated from four animals. Threeday wound (C) or lung (D) tissue isolated from the same individual mouse was cultured ex vivo in the absence (w/o IL-4) or presence (+IL-4) of 10 $\mathrm{ng} / \mathrm{mL}$ of IL- 4 for 24 hours. Sections were double stained using the macrophage-specific F4/80 (red signals) and an anti-Ym1 antibody (brown signals). Arrowheads indicate Ym1-free wound macrophages (C) or Ym1-expressing lung macrophages (D). Bars indicate means \pm SD. $* * P<0.01$ (Student's unpaired $t$-test) compared with nonstimulated cells (-). 

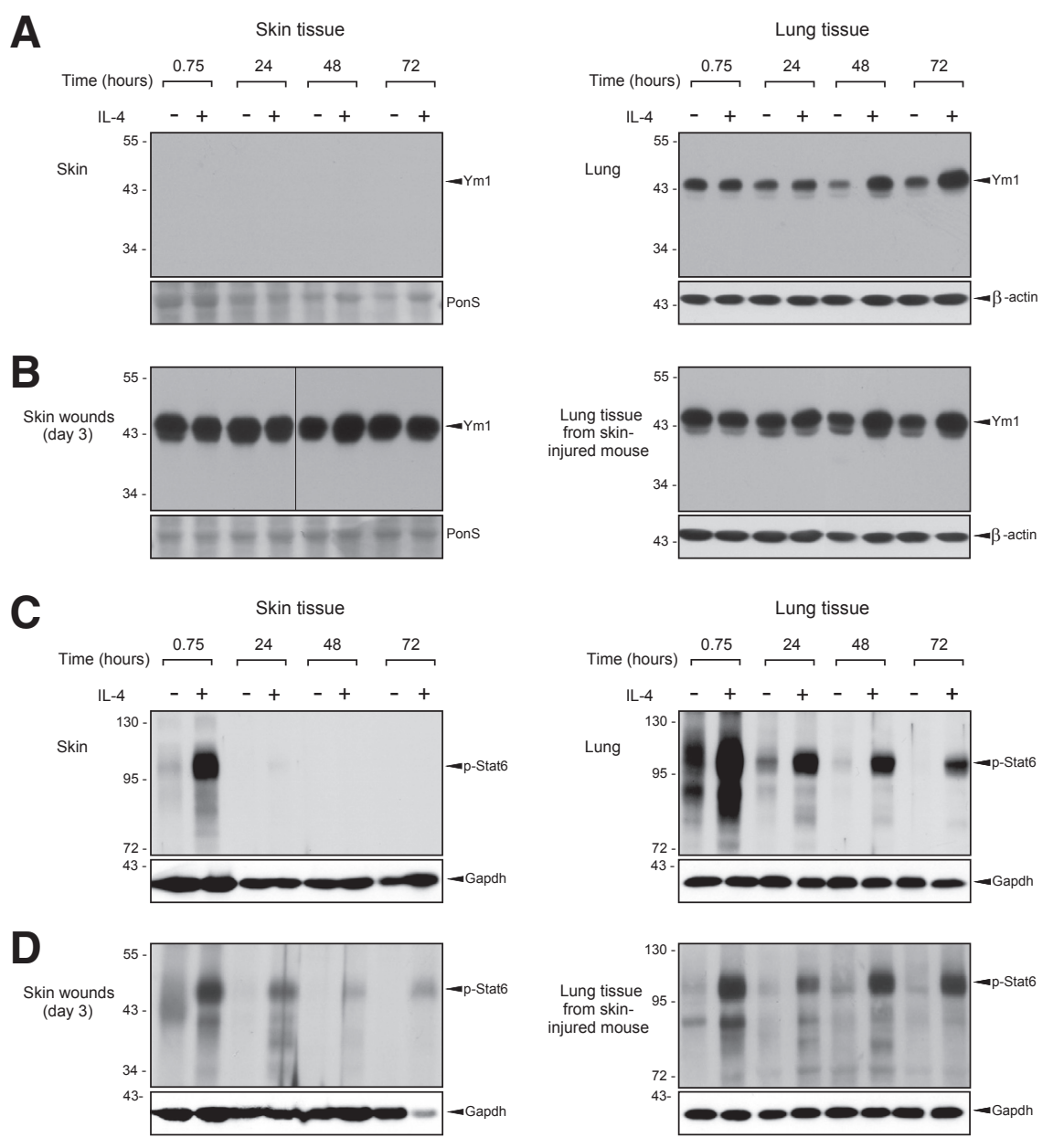

Figure 7 IL-4 activates Stat6 in ex vivocultured skin, wound, and lung tissue. Skin and lung tissue was isolated from nonwounded wildtype mice (A and $\mathbf{C})$. Skin wounds and lung tissue were isolated from the same individual mice on day 3 after injury (B and $\mathbf{D})$. All the tissues were cultured ex vivo in the absence or presence of $10 \mathrm{ng} / \mathrm{mL}$ of IL-4 for the indicated periods. Skin and lung tissues from nonwounded mice were analyzed by immunoblot for Ym1 (A) or activated Stat6 (p-Stat6) (C). Skin wounds and lung tissue from wounded mice were analyzed by immunoblot for Ym1 (B) or p-Stat6 (D). Ponceau S (PonS) staining, $\beta$-actin, and GAPDH were used to control loading. toward an alternatively activated wound-healing macrophage phenotype. Most important, considerable events known to drive an alternative maturation of macrophages could not be substantiated in a murine wound model: essentially, the presence of IL-4 and the subsequent activation of Stat6 in the cells. ${ }^{4}$ Based on the widely accepted notion of Ym1 as a marker of alternative activation of macrophages, ${ }^{7-9,11}$ Lucas et $\mathrm{al}^{12}$ proposed such an alternative activation of woundhealing macrophages in acute wounds. However, as IL-4 is missing in acute murine wounds, ${ }^{4}$ one has to be cautious to oversimplify the easy presence of alternative macrophage markers (such as Ym1) to define wound-healing macrophage phenotypes. This notion is further supported by a most recent finding showing that simultaneous adenosine $\mathrm{A}_{2 \mathrm{a}}$ receptor and LPS-mediated Toll-like receptor stimulation was also capable of driving an alternative activation of cultured peritoneal macrophages. Again, that adenosine/LPS-mediated switch toward an alternative macrophage polarization was independent of IL-4 signaling and characterized by the absence of Ym1 and resistin-like molecule $\alpha^{33}$

In addition, impaired wound tissue has not yet been analyzed with respect to standard markers in macrophage maturation. $^{7,8}$ Therefore, it was interesting to observe elevated levels of Ym1 mRNA and protein particularly in nonhealing wound tissue, as Ym1 is currently evaluated and used to define an IL-4-triggered wound-healing macrophage phenotype with beneficial properties in wound healing. ${ }^{12}$ Herein, we confirmed the absence of IL-4 and activation of Stat6 from acute wounds in mice. ${ }^{4}$ Thus, our finding revealed wound conditions with elevated Ym1 and Arg-1 levels in the absence of the inducing cytokine. This notion was further strengthened in Stat6-deficient mice. ${ }^{34}$ The mice revealed normal expression of Ym1 and Arg-1 in wound tissue. That finding again implicates that these established markers of alternative macrophage activation $^{10,11}$ do not functionally couple to IL-4/Stat6 signaling in wound healing.

These data suggest infiltrating neutrophils as the main cellular source of Ym1 protein in wounds. Early studies on $\mathrm{Ym} 1$ have shown prominent expression of the protein in $\mathrm{F} 4 /$ $80^{(-)}$myeloid precursor cells of hematopoietic tissues, particularly in fetal and adult liver, spleen, and bone marrow, indicating that the protein was predominantly expressed in nonmonocytic cells. ${ }^{35}$ In accordance with this report, we first observed a significant increase in $\mathrm{Ym} 1^{(+)} / \mathrm{Ly}-6 \mathrm{G}^{(+)}$cells in the bone marrow of wounded mice, representing neutrophil precursors. ${ }^{36}$ This is consistent with observations from a genetic mouse model of chronic granulomatous disease, 
A

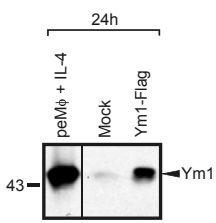

B

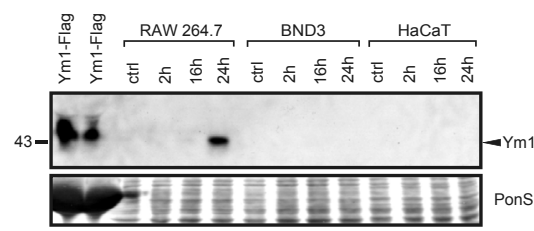

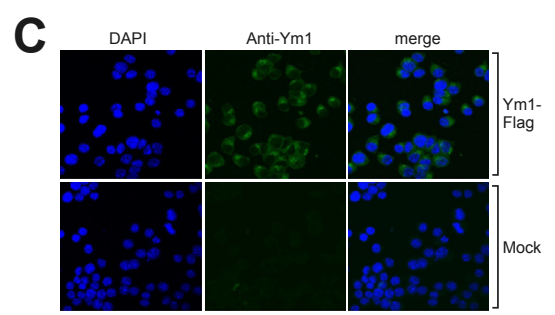

E

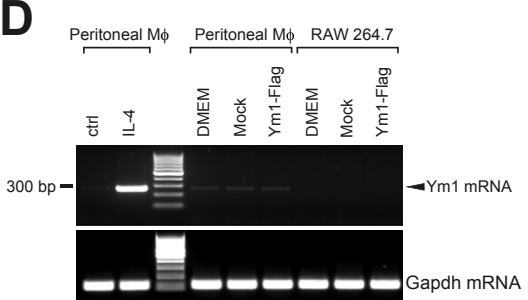

$\mathbf{F}$

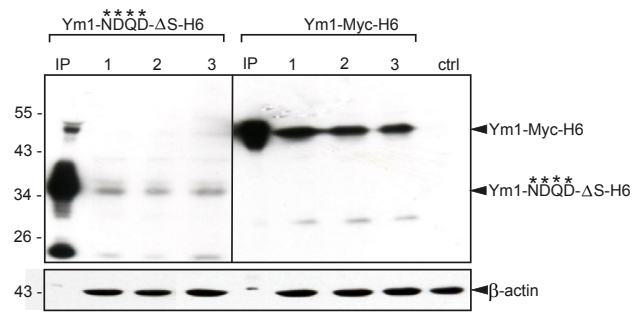

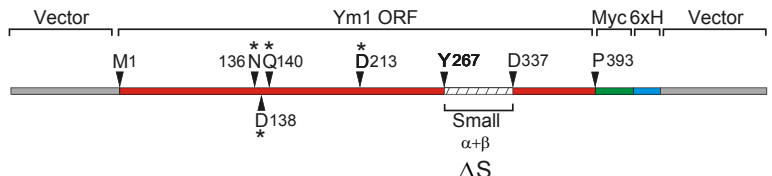

$\Delta S$

Figure 8 Uptake of Ym1 is restricted to macrophages and is dependent on protein structure. A: Analysis of conditioned media from mock- and Ym1Flag-transfected HEK cells. IL-4-treated $(10 \mathrm{ng} / \mathrm{mL})$ primary peritoneal macrophages served as a positive control for Ym1 detection. B: RAW $264.7 \mathrm{macro-}$ phages, endothelial cells (BND3), and keratinocytes (HaCaT) were incubated with conditioned medium from Ym1-Flag-transfected HEK cells for 24 hours for the indicated periods. Cellular lysates were analyzed by immunoblot for the intracellular presence of Ym1. Twenty-five microliters of conditioned medium from Ym1Flag-transfected HEK cells (Ym1-Flag) served as a positive control. Ponceau S (PonS) staining was used to control loading. C: RAW 264.7 macrophages were incubated with conditioned medium from Ym1-Flag - or mock-transfected HEK cells for 24 hours. Ym1 protein uptake was assessed by immunofluorescence. Nuclei were stained with DAPI. D: RT-PCR analysis of Ym1 mRNA in primary peritoneal and RAW 264.7 macrophages. Primary macrophages were left unstimulated (ctrl) or were treated with $10 \mathrm{ng} / \mathrm{nL}$ of IL-4 for 24 hours as a positive control. Primary and RAW 264.7 macrophages were incubated with fresh medium [Dulbecco's modified Eagle's medium (DMEM)] or conditioned medium from mock- or Ym1-Flag-transfected HEK cells for 24 hours as indicated. GAPDH mRNA served to control equal loading. E: Scheme of the expression vector for recombinant Ym1 protein. The Ym1 open reading frame (ORF) is shown in red, mutated amino acid residues are marked by asterisks, and the positions of the deleted small $\alpha+\beta$ domain $(\Delta S)$ and the Myc and $6 x$ His tags are indicated. F: Primary peritoneal macrophages were incubated with purified Myc-tagged mutated Ym1 $\left(Y m 1-N^{*} D^{*} Q^{*} D^{*}-\Delta S-H 6\right)$ or Myc-tagged wild-type Ym1 (Ym1-Myc-H6) protein for 24 hours. Cells were washed intensively. Cellular lysates were analyzed by immunoblot for the intracellular presence of recombinant Ym1 using an anti-Myc antibody. Three experiments (1-3) are shown. $\beta$-Actin was used to control loading. G: Primary macrophages were stimulated with increasing concentrations of LPS in the absence or presence of purified recombinant Ym1-Myc protein for 24 hours as indicated. Cellular lysates were assessed for inducible nitric oxide synthase (iNOS) and cyclooxygenase 2 (Cox-2) expression by immunoblot. $\beta$-Actin was used to control loading. IP, immunoprecipitation; peM $\phi$, peritoneal macrophages.

where peritoneal and bone marrow-derived neutrophils expressed Ym1 at concentrations one order of magnitude higher compared with macrophages at the assessed sites of inflammation. ${ }^{14}$

This observation was also true for skin wounds (this study). A skin wound is a well-known site of acute inflammation, ${ }^{37}$ which is often prolonged and aggravated in disturbed, nonhealing conditions. ${ }^{25,28}$ The acute phase of cutaneous wound inflammation is paralleled by the presence of the neutrophil infiltrate. Neutrophils disappear as soon as the wound inflammation is resolved but markedly persist during impaired conditions. ${ }^{25,28,31}$ The observed Ym1 expression kinetics in normal and late diabetes-disturbed wounds perfectly matched the presence of neutrophils, and we could allocate the woundderived Ym1 mRNA and protein to infiltrating wound neutrophils but not to macrophages (this study). Here, it is tempting to argue that only those infiltrating $\mathrm{F} 4 / 80^{(+)}$woundhealing macrophages became positive for $\mathrm{Ym} 1$ protein in wound tissue that had been in close spatial vicinity to the observed $\mathrm{Yml}^{(+)}$wound neutrophils. This phenomenon thereby generated a visible gradient of $\mathrm{Yml}^{(+)}$wound macrophages, which peak in numbers in the center of the wound, essentially where the neutrophils crowded together.

Thus, two scenes might provide explanations for the observed $\mathrm{Ym}^{(+)}$wound-healing macrophage gradient in the absence of an IL-4/Stat6 activation in wounds. First, wound macrophages might acquire $\mathrm{Ym} 1$ by engulfment of apoptotic neutrophils at the wound site. ${ }^{38-40}$ The selective uptake of a secreted $\mathrm{Ym} 1$ protein might represent the second alternative to explain $\mathrm{Ym}^{(+)}$wound-healing macrophages in the absence of IL-4 at wounded sites. A 21 -amino acid signal peptide for secretion ${ }^{15,24}$ and the 
capability of neutrophils to secrete $\mathrm{Ym} 1^{14}$ might argue for this notion. The selective uptake of the recombinant $\mathrm{Ym} 1$ protein into cultured macrophages, but not endothelial and epithelial cells, supports this hypothesis. The subsequent mutation of amino acid residues $\mathrm{N}^{136}, \mathrm{D}^{138}, \mathrm{Q}^{140}$, and $\mathrm{D}^{213}$ and deletion of the small $\alpha+\beta$ domain $(\Delta S)$, necessary for a correctly folded and functional $\mathrm{Ym} 1$ protein, ${ }^{24}$ abrogated the uptake of recombinant $\mathrm{Ym} 1$ protein into cultured macrophages and speaks in favor of a specific uptake of Ym1 protein into macrophages. Recombinant Ym1 reduced a low-grade inflammatory response from macrophages to LPS. Thus, Ym1 protein from exogenous neutrophil sources might, therefore, contribute to antiinflammatory changes in wound-healing macrophages in response to wound neutrophils. ${ }^{38-40}$

The presented data suggest that the activation of woundhealing macrophages and expression of the Ym1 marker protein were not functionally connected to the IL-4-/Stat6dependent signaling pathway, despite its accepted role in alternative activation of macrophages. Thus, the paradigm of an alternative activation of regenerative wound-healing macrophages might not provide a solid basis to categorize a common repair phenotype of the wound-healing macrophage.

\section{Acknowledgments}

We thank Drs. Christoph Schürmann and Kerstin EngelmannPilger for helpful assistance in manuscript preparation.

\section{References}

1. Leibovich SJ, Ross R: The role of the macrophage in wound repair. a study with hydrocortisone and antimacrophage serum. Am J Pathol 1975, 78:71-100

2. Brancato SK, Albina JE: Wound macrophages as key regulators of repair. origin, phenotype and function. Am J Pathol 2011, 178:19-25

3. Geissmann F, Jung S, Littman DR: Blood monocytes consist of two principal subsets with distinct migratory properties. Immunity 2003, 19:71-82

4. Daley JM, Brancato SK, Thomay AA, Reichner JS, Albina JE: The phenotype of murine wound macrophages. J Leukoc Biol 2010, 87: 59-67

5. Nahrendorf M, Swirski FK, Aikawa E, Stangenberg L, Wurdinger T, Figueiredo JL, Libby P, Weissleder R, Pittet MJ: The healing myocardium sequentially mobilizes two monocyte subsets with divergent and complementary functions. J Exp Med 2007, 204:3037-3047

6. Ishida Y, Gao JL, Murphy PM: Chemokine receptor CX3CR1 mediates skin wound healing by promoting macrophage and fibroblast accumulation and function. J Immunol 2008, 180:569-579

7. Mosser DM, Edwards JP: Exploring the full spectrum of macrophage activation. Nat Rev Immunol 2008, 8:958-969

8. Gordon S: Alternative activation of macrophages. Nat Rev Immunol 2003, 3:23-35

9. Raes G, De Baetselier P, Noël W, Beschin A, Brombacher F, Hassanzadeh Ghassabeh G: Differential expression of FIZZ1 and Ym1 in alternatively versus classically activated macrophages. J Leukoc Biol 2002, 71:597-602

10. Raes G, Van den Bergh R, De Baetselier P, Hassanzadeh Ghassabeh G: Arginase-1 and Ym1 are markers for murine, but not human, alternatively activated myeloid cells. J Immunol 2005, 174:6561
11. Welch JS, Escoubet-Lozach L, Sykes DB, Liddiard K, Greaves DR, Glass CK: Th2 cytokines and allergic challenge induce Ym1 expression in macrophages by a STAT6-dependent mechanism. J Biol Chem 2002, 277:42821-42829

12. Lucas T, Waisman A, Ranjan R, Roes J, Krieg T, Müller W, Roers A, Eming SA: Differential roles of macrophages in diverse phases of skin repair. J Immunol 2010, 184:3964-3977

13. Martinez FO, Helming L, Gordon S: Alternative activation of macrophages: an immunologic functional perspective. Annu Rev Immunol 2009, 27:451-483

14. Harbord M, Novelli M, Canas B, Power D, Davis C, GodovacZimmermann J, Roes J, Segal AW: Ym1 is a neutrophil granule protein that crystallizes in p4 $7^{\text {phox }}$-deficient mice. J Biol Chem 2002, 277: $5468-5475$

15. Chang NCA, Hung SI, Hwa KY, Kato I, Chen JE, Liu CH, Chang AC: A macrophage protein, Ym1, transiently expressed during inflammation is a novel mammalian lectin. J Biol Chem 2001, 276: 17497-17506

16. Stallmeyer B, Kämpfer H, Kolb N, Pfeilschifter J, Frank S: The function of nitric oxide in wound repair: inhibition of inducible nitric oxide-synthase severely impairs wound reepithelialization. J Invest Dermatol 1999, 113:1090-1098

17. Frank S, Stallmeyer B, Kämpfer H, Kolb N, Pfeilschifter J: Nitric oxide triggers enhanced induction of vascular endothelial growth factor expression in cultured keratinocytes $(\mathrm{HaCaT})$ and during cutaneous wound repair. FASEB J 1999, 13:2002-2014

18. Thakur ML, Li J, Chandy B, John EK, Gibbons G: Transient neutropenia: neutrophil distribution and replacement. J Nucl Med 1996, $37: 489-494$

19. Frank S, Stallmeyer B, Kämpfer H, Kolb N, Pfeilschifter J: Leptin enhances wound re-epithelialization and constitutes a direct function of leptin in skin repair. J Clin Invest 2000, 106:501-509

20. Goren I, Müller E, Schiefelbein D, Christen U, Pfeilschifter J, Mühl H, Frank S: Systemic anti-TNFalpha treatment restores diabetes-impaired skin repair in ob/ob mice by inactivation of macrophages. J Invest Dermatol 2007, 27:2259-2267

21. Boukamp P, Petrussevska RT, Breitkreutz D, Hornung J, Markham A, Fusenig NE: Normal keratinization in a spontaneously immortalized aneuploid human keratinocyte cell line. J Cell Biol 1988, 106:761-771

22. Chomczynski P, Sacchi N: Single-step method of RNA isolation by acid guanidinium thiocyanate-phenol-chloroform extraction. Anal Biochem 1987, 162:156-159

23. Goren I, Müller E, Schiefelbein D, Gutwein P, Seitz O, Pfeilschifter J, Frank S: Akt1 controls insulin-driven VEGF biosynthesis from keratinocytes: implications for normal and diabetes-impaired skin repair in mice. J Invest Dermatol 2009, 129:752-764

24. Sun YJ, Chang NCA, Hung SI, Chang AC, Chou CC, Hsiao CD: The crystal structure of a novel mammalian lectin, Ym1, suggests a saccharide binding site. J Biol Chem 2001, 276:17507-17514

25. Goren I, Kämpfer H, Podda M, Pfeilschifter J, Frank S: Leptin and wound inflammation in diabetic ob/ob mice: differential regulation of neutrophil and macrophage influx and a potential role for the scab as a sink for inflammatory cells and mediators. Diabetes 2003, 52: $2821-2832$

26. Kämpfer H, Schmidt R, Geisslinger G, Pfeilschifter J, Frank S: Wound inflammation in diabetic ob/ob mice: functional coupling of prostaglandin biosynthesis to cyclooxygenase-1 activity in diabetes-impaired wound healing. Diabetes 2005, 54:1543-1551

27. Kämpfer H, Bräutigam L, Geisslinger G, Pfeilschifter J, Frank S: Cyclooxygenase-1-coupled prostaglandin biosynthesis constitutes an essential prerequisite for skin repair. J Invest Dermatol 2003, 120:880-890

28. Wetzler C, Kämpfer H, Stallmeyer B, Pfeilschifter J, Frank S: Large and sustained induction of chemokines during impaired wound healing in the genetically diabetic mouse: prolonged persistence of neutrophils and macrophages during the late phase of repair. J Invest Dermatol 2000, 115:245-253 
29. Austyn JM, Gordon S: F4/80, monoclonal antibody directed specifically against the mouse macrophage. Eur J Immunol 1981, 11:805-815

30. McKnight AJ, Macfarlane AJ, Dri P, Turley L, Willis AC, Gordon S: Molecular cloning of F4/80, a murine macrophage-restricted cell surface glycoprotein with homology to the G-protein-linked transmembrane 7 hormone receptor family. J Biol Chem 1986, 271:486-489

31. Buiting AM, van Rooijen N: Liposome mediated depletion of macrophages: an approach for fundamental studies. J Drug Target 1994, 2 : $357-362$

32. Goren I, Allmann N, Yogev N, Schürmann C, Linke A, Holdener M, Waisman A, Pfeilschifter J, Frank S: A transgenic mouse model of inducible macrophage depletion: effects of diphtheria toxin-driven lysozyme M-specific cell lineage ablation on wound inflammatory, angiogenic, and contractive processes. Am J Pathol 2009, 175: $132-147$

33. Ferrante CJ, Pinhal-Enfield G, Elson G, Cronstein BN, Hasko G, Outram S, Leibovich SJ: The adenosine-dependent angiogenic switch of macrophages to an M2-like phenotype is independent of interleukin-4 receptor alpha (IL4Ra) signaling. Inflammation 2013, 36:921-931

34. Kaplan MH, Schindler U, Smiley ST, Grusby MJ: Stat6 is required for mediating responses to IL-4 and for development of Th2 cells. Immunity 1996, 4:313-319
35. Hung SI, Chang AC, Kato I, Chang NCA: Transient expression of Ym1, a heparin binding lectin, during developmental hematopoiesis and inflammation. J Leukoc Biol 2002, 72:72-82

36. Daley JM, Thomay AA, Connolly MD, Reichner JS, Albina JE: Use of Ly6G-specific monoclonal antibody to deplete neutrophils in mice. J Leukoc Biol 2008, 83:64-70

37. Martin P: Wound healing: aiming for perfect skin regeneration. Science 1997, 276:75-81

38. Fadok VA, Bratton DL, Konowal A, Freed PW, Westcott JY, Henson PM: Macrophages that have ingested apoptotic cells in vitro inhibit proinflammatory cytokine production through autocrine/paracrine mechanisms involving TGF-beta, PGE2, and PAF. J Clin Invest 1998, 101:890-898

39. McDonald PP, Fadok VA, Bratton D, Henson PM: Transcriptional and translational regulation of inflammatory mediator production by endogenous TGF-beta in macrophages that have ingested apoptotic cells. J Immunol 1999, 163:6164-6172

40. Peters T, Sindrilaru A, Hinz B, Hinrichs R, Menke A, Al-Azzeh EA, Holzwarth K, Oreshkova T, Wang H, Kess D, Walzog B, Sulyok S, Sunderkötter C, Friedrich W, Wlaschek M, Krieg T, ScharffetterKochanek K: Wound-healing defect of CD18(-/-) mice due to a decrease in TGF-beta1 and myofibroblast differentiation. EMBO J 2005, 24:3400-3410 